\section{Public Health Significance of Foodborne Helminthiasis: A Systematic Review}

\author{
Mahendra Pal ${ }^{1 *}$, Yodit Ayele ${ }^{2}$, Angesom Hadush ${ }^{3}$, Pooja Kundu ${ }^{4}$ and Vijay J Jadhav ${ }^{4}$ \\ ${ }^{1}$ Narayan Consultancy on Veterinary Public Health, 4 Aangan, Jagnath Ganesh Dairy Road, Anand-38001, India \\ ${ }^{2}$ Department of Animal Science, College of Agriculture and Natural Resources, Bonga University, Post Box No.334, Bonga, Ethiopia \\ ${ }^{3}$ Department of Animal Production and Technology, College of Agriculture and Environmental Sciences, Adigrat University, P.O. Box 50, Adigrat, Ethiopia \\ ${ }^{4}$ Department of Veterinary Public Health and Epidemiology, College of Veterinary Sciences, LUVAS, Hisar-125004, India
}

*Corresponding author: Mahendra Pal, Narayan Consultancy on Veterinary Public Health and Microbiology, 4 Aangan, Jagnath Ganesh Dairy Road, Anand-388001, Gujarat, India, E-mail: palmahendra2@gmail.com

Received date: December 18, 2017; Accepted date: January 19, 2018; Published date: January 25, 2018

Copyright: @2017 Pal M, et al. This is an open-access article distributed under the terms of the Creative Commons Attribution License, which permits unrestricted use, distribution, and reproduction in any medium, provided the original author and source are credited.

\begin{abstract}
Foodborne diseases, caused by biological as well as chemical agents, have an impact in both developing and developed nations. The foodborne diseases of microbial origin are acute where as those caused by chemical toxicants are resulted due to chronic exposure. Amongst various microbial agents of foodborne diseases, parasitic infections have been recently identified as an important public health problems having considerable economic impact in terms of morbidity, loss of productivity, and health care costs. The risk factors for acquiring foodborne helminthic infections are overcrowding, unhygienic living conditions, scarcity of water, contaminated food and water, under nutrition and other poverty-related factors. According to the World Health Organization, there are 32 important foodborne pathogens, of which 10 are helminthes. Several foodborne and waterborne helminthic diseases such as echinococcosis, fascioliasis, taeniasis, cysticercosis, diphyllobothriosis, capillariasis, and ascariasis have shown increased trend in the last decades. Majority of the mentioned parasites have no successful detection and controlling mechanisms. Immunization of food animals against foodborne parasites in certain conditions is very limited because there is generally a lack of vaccines for foodborne parasites. Mass chemotherapy has been found most suitable approach to reduce the impact of helminthic diseases. In addition, education of consumers, food handlers and food producers with respect to personal hygiene is an essential component of food safety. Further, there is a need to strengthen veterinary services in order to reduce the occurrence of such diseases in livestock population. Emphasis should be given to undertake further studies on the diagnostic techniques and epidemiology of foodborne helminthic diseases.
\end{abstract}

Keywords: Emerging pathogen; Foodborne infection; Helminths; Parasites; Public health; Zoonoses

\section{Introduction}

Foodborne diseases, which involve multiple etiologies, are growing public health concern throughout the world. There are over 200 diseases spread through foods. Such diseases are playing a significant role in causing economic losses through serious proportion of morbidity and mortality rate worldwide. Since the foodborne disease causing organisms can also enter through other routes and produce disease, it is difficult to determine the exact mortality associated with foodborne illnesses [1]. Globally, thirty two important foodborne diseases are reported, out of which 10 are caused by helminths. According to the global reports in 2010, 600 million foodborne illnesses and 420,000 deaths were recorded worldwide [2]. Majority of the illnesses caused by these hazards are mild and self-limiting. However, significant morbidity and mortality cases can occur in highrisk groups including infants, young children, the elderly and the immunocompromised persons [3]. Amongst the various types of foodborne diseases, parasitic diseases are often associated with poverty-related factors of developing countries, such as poor hygiene and sanitation, contaminated food and water, overcrowding, and malnutrition. More than one-quarter of the world's population mainly the people from developing countries, is thought to be chronically infected with intestinal parasites [4]. Intestinal parasitic infections, as in many developing counties, are common in Ethiopia and cause serious public health problems such as malnutrition, anemia, and growth retardation as well as higher susceptibility to other infections [4]. It is estimated that one person in every four harbors parasitic worms. The parasitic infections prevalent in the developing tropical nations are spreading to the developed non-tropical countries by the tourists, soldiers, or immigrants [5].

Overall, foodborne parasitic diseases, excluding enteric protozoa, caused an estimated 23.2 million cases and 45,927 deaths annually [6]. Foodborne and waterborne parasitic diseases occur in sporadic as well as in epidemic form resulting into high morbidity and mortality in susceptible individuals $[7,8]$. It is acknowledged that foodborne parasitic infections affect many millions of people worldwide [9].

Helminth parasites have unique features, which often make them remarkably suited for survival in the environment that makes them different from bacteria and viruses. These special features make detection and control of parasites difficult. Generally, these parasites cause severe public health problems and considerable economic losses in the livestock sector [10]. Provided that these parasites have such an impact, the two key components of prevention and control are improved sanitation mainly avoidance of food and water contamination and health education about the modes of transmission of infections. The survey on prevalence of intestinal parasites provides 
information concerning the burden of parasites in a community, enabling to predict risks for communities under consideration and decisions to be made as to the need for intervention [4]. Therefore, the objective of this systematic review is to delineate the important foodborne helminths and their public health implications.

\section{Foodborne Zoonotic Helminthic Diseases}

\section{Foodborne zoonotic cestode infections}

Diphyllobothriosis: Diphylobothiosis is an important fish borne zoonotic helminthosis, which is caused by tapeworm belonging to the genus Diphyllobothrium. It is reported that around 20 million people are infected worldwide by this parasite. To date, approximately 50 species have been described within the Diphyllobothrium genus but only 13 have been reported as human pathogens; the most frequent species being $D$. latum, D. nihonkaiense and D. dendriticum [11]. Diphyllobothrium latum is the largest tapeworm of fish eating mammals, which is transmitted to humans through consumption of raw or undercooked fish. These parasites are commonly found in countries that have peoples with fish consumption behavior including Europe, North and South America and Asia. Prolonged infection causes different types of anemia, such as macrocytic, hypocromic, and pernicious anaemia resulting from parasite competition that makes vitamin B12 unavailable to the host [12]. Intestinal infection with $D$. latum is usually asymptomatic. In some cases, mild gastrointestinal obstruction, rarely diarrhea and abdominal pain, and occasionally leukocytosis with eosinophilia are present. Studies performed in Finland indicated that anaemia occurs in approximately $2 \%$ of the patients [13].

Echinococcosis: Echinococcosis, an emerging and re-emerging cyclozoonosis, is primarily caused Echinococcus granulosus. The infection due to E. granulosus is reported from 77 countries including 20 in the Asia Pacific region. Globally, E. granulosus is responsible for 100,000 cases annually. Humans acquire the infection by accidental ingestion of food and water contaminated with eggs of the genus Echinococcus. Raw vegetables and fruits can be contaminated by theses eggs resulting in a foodborne infection. From public health perspective, four species including Echinococcus granulosus (causes cystic echinococcosis), Echinococcus multilocularis (causes alveolar echinococcosis), Echinococcus oligarthus and Echinococcus vogeli (causes polycystic echinococcosis) have been considered as important [14].

Liver is the most common site for the hydatid cyst development, and humans are accidental intermediate and dead end host for the parasite. The cyst can be found in other organs, such as the lungs, spleen, kidneys, heart, bone, and central nervous system. Cysts may stay for years before showing any clinical signs and may reach up to 10 $\mathrm{cm}$ in diameter per year. Serious allergic or immunological reactions of mild to fatal anaphylaxis can occur due to a sudden cyst rupture and release of its contents [14,15]. Echinococcus granulosus, a dog tapeworm, is known to occur on all the continents. High prevalence of Echinococcus in wild and domestic animals is associated with disease incidence in humans. Hydatid cysts can cause pain in the upper abdominal region, hepatomegaly, cholestasis, billiary cirrhosis, portal hypertension, ascites, fever, anemia, weight loss, and pleural pain $[16,17]$.

Taeniasis/Cysticercosis: Taeniasis is an important meat borne cyclozoonosis, and is recorded from many countries of the world.
Different life stages of Taenia species causes distinct diseases of cysticercosis and taeniasis in animals and humans, respectively. The two most commonly known tapeworms in human beings are $T$. saginata and T. solium. The life cycle of Taenia saginata depends on the association between humans and cattle and Taenia solium between humans and pigs. Cysticercosis is an infection caused mainly by ingestion of taenid eggs. Practices such as improved animal husbandry, sanitation, and better meat inspection, over the past decades, has substantially decreased the incidence of cysticercosis worldwide [18]. Cysticercosis both in cattle and pigs may result in reduced carcass value of partial or full condemnation. In USA, the economic losses due to cysticercosis in feedlot cattle was estimated US Dollar 500,000 to 800,000 . Taenid eggs and T. solium cysts have been found in the vegetables and in dog meat sold in Hanoi, respectively [19,20]. Eating raw/pickled meat and agricultural use of human wastewater as fertilizer may be a major risk factor for transmission and spread of cysticercosis/teaniasis [21]. Ingestion of $T$. solium eggs give rise to the larval stage of the pork tapeworm and causes the clinical syndrome of cysticercosis. As these cysts may lodge in the brain, they cause neurocysticercosis [22]. Neurocysticercosis is one of the serious complications of this infection in humans. It is pertinent to mention that $26.3 \%$ to $53.8 \%$ of adult epilepsy cases in the developing world including India and Latin America are due to neurocysticercosis. It is also becoming more common in the developed world because of increased migration of people with these diseases and frequent travel to the endemic countries [23].

The common signs caused by these diseases are headache, vomition, dimness of vision/visual disturbances, acute supportive dacryoedinitis, and cystic nodules on neck, focal neurologic symptoms, pain due to localized skeletal muscle nodules, seizures, and convulsions. Cysticercus may present in anterior chamber of eye or outer part of the eyelid. Ophthalmic cysticercosis (intraocular) manifests symptoms, such as proptosis, diplopia, and loss of vision while extraocular cyst resembles slow growing tumour or nodule with focal inflammation. Intestinal taeniasis may be associated with abdominal discomfort, irritation of intestinal mucosa, obstruction, nausea, weight loss, and anal pruritis [24].

Infection of Taenia solium is present in tropical countries but it is eradicated in most developed countries, mainly due to general socioeconomic development and intensification of pig husbandry systems. It is associated with poverty and unhygienic condition such as allowing pigs to have access to human feces. Global estimation has showed that about 66 millions of persons worldwide are infected with T. solium, mainly in Sub-Saharan Africa, Latin America and South and SouthEast Asia. The rapid expansion of smallholder extensive swine production has resulted in significant increase of cysticercosis in pigs and humans. However, the beef tapeworm is found in developed as well as in developing countries. Taenia saginata infection in humans has been reported from 45 countries and 45-60 million people are affected. The prevalence of $T$. saginata infection in India is low. Cysticerci in meat can easily destroyed by applying high temperatures on food and hygienic practices, which readily affect the transmission $[25,26]$.

\section{Foodborne zoonotic nematode infections}

Ascariasis: The common intestinal roundworm parasite infecting an estimated one-quarter of the world's population is caused by Ascaris lumbricoides [27]. Newborn babies may become infected within months after birth in areas without adequate hygiene. Stunted growth, 
diarrheal infections, and early childhood mortality may happen due to subsequent growth of the worm. In adults, there is no exhibition of symptoms except irritation of intestinal lining and interference with the absorption of fats and protein. However, in some cases, it may cause more severe infections in the liver or lungs.

Eggs passed out with feces may be ingested by the same or another person through drinking contaminated water, eating with dirty hands, or by consumption of uncooked vegetables that have been fertilized with contaminated human wastes. Following ingestion, eggs hatch in the intestine and worms may migrate to lungs or liver before returning to the intestine for maturation. There are techniques developed for detection and enumeration of Ascaris eggs on vegetables, and it may be useful for screening of contaminated fresh vegetables before consumption $[28,29]$.

Capillariasis: Capillaria philippinensis occurs over a wide geographical area extending from Philippines, where infection occurs as small epidemics along rivers, to Egypt where individual cases have been reported [13]. There are more than 250 species of Capillaria identified, and only three, $C$. philippinensis, $C$. hepatica, and $C$. aerophilia, are known to infect humans. Out of these three, $C$. philippinensis is the only species with great significance and potentially an emerging zoonotic helminth. Cappilaria philippinensis infection was first identified and described in the rural communities of Philippines in 1965 [30].

The parasite infects humans through eating uncooked small fresh water fish and it is enzootic in fish eating birds. Fishes primarily contract the infection from the contaminated water by feces of infected humans or birds. However, the role of other fish-eating mammals in transmission of the infection is not clearly defined. Amongst the impacts of the parasite, chronic malabsorption syndrome has occasionally been reported to be fatal. In untreated cases, progressive small bowel dysfunction marked by diarrhea, abdominal pain and weight loss may be developed due to increment of parasite load. The infection is fatal unless appropriate anthelmintic chemotherapy is employed. It can be prevented through public education and can be treated with mebendazole. The parasite burden is greatly reduced in areas where the practice of consuming undercooked fish has been avoided [13].

Gnathostomiasis: Gnathostomiasis is caused by different species of the nematode Gnathostoma, and it is an emerging foodborne zoonotic disease [31]. It is being observed with increasing frequency in countries where it is not endemic, and should be regarded as another emerging imported disease. It is a parasite of wild carnivores, cats, and dogs. Previously, most of these cases were reported from Southeast Asia, particularly Thailand and Japan, because of the dietary habits. However, in recent years, it is an increasing public health problem in Central and South America [32]. Gnathostoma spinigerum and other species are also found in India, Pakistan, the Middle East, Mexico, Southeast Asia, Japan, and Australia. In India, there are several reports of such cases from different areas since first case of ocular gnathostomiasis was reported in 1945 [31].

The disease is acquired by consuming uncooked meat of fish, shrimp, crab, crayfish, frog, or chicken infected with the third stage larva [31,32]. The immature worms may be found in any part of the body such as mouth, eye, pharynx, respiratory tract, urinary bladder, intestine or anus [33]. The important clinical symptoms of gnathostomiasis are emanated from the inflammatory reaction caused by the migrating larvae. These appear commonly in the upper extremities, shoulder, neck, and other parts of the body. Treatment for superficially occurring larvae may be surgical removal [31].

Trichinellosis: Trichinellosis is a foodborne helminthic zoonosis, which occurs by taking encysted larvae of the genus Trichinella during consumption of contaminated pork. This disease is mainly caused by Trichinella spiralis, which is commonly found in pigs and wild boars [34]. In Europe, trichinellosis has been described as an emerging or reemerging disease during the past decades. In 2008, morbidity due to trichinellosis doubled in Russia. According to a report of the European Food Safety Authority, trichinellosis caused 779 human cases in EU Member States during 2007 [35]. The infection due to Trichinella species has been detected both in domestic and wild animals in every continent of the world except Antarctica [36].

Trichinella larvae of encysted in muscular tissue of domestic or wild animal, causes infection in humans; however, domestic pigs are major source of human infection worldwide [37]. The organs of the pig most heavily involved are tongue, masseter, diaphragm, and intercostal muscles [35]. However, there are reports in which meat sourced from wild boars, and horses have involved in outbreaks within the past decades. Cultural food practices including the consumption of raw or undercooked meat has contributed for the occurrence of trichinosis in humans. The global average of annual incidence of the disease in humans is around 10,000 cases having a mortality rate of about $0.2 \%$. However, this record is thought to be underreported in many nations because of lack of adequate awareness and absence of appropriate diagnostic facilities [37].

Man is the accidental host and dead end of the parasite. Heavy infections due to this helminth in humans may induce an illness having three clinical phases, which are intestinal, muscle invasion, and convalescent. In more severe cases, difficulties with coordinating movements as well as heart and breathing problems may occur. Cardio-neurological syndrome, nephritis, and pneumonia are the occasional life threatening manifestations. A small proportion of cases die from Trichinella infection [35]. Trichinosis has both economic and public health implications associated with porcine production and safety. As far as zoonotic importance of the infection is concerned, many countries have focused on control of this helminth by eliminating it from the food chain.

\section{Foodborne zoonotic trematode infections}

Clonorchiosis and Opisthorchiosis: Clonorchis sinensis, Opisthorchis viverrini, and Opisthorchis felineus, the human liver flukes, are parasites of fish-eating mammals, particularly in Asia and Europe. In highly endemic areas, such as north-east Thailand, the prevalence of $O$. viverrini may reach up to $90 \%$ [38]. Clonorchis sinensis is highly prevalent in the People's Republic of China, Taipei China, and Vietnam; and still occurs in Japan and the Korean peninsula. The principal animal hosts are cats, dogs, pigs, rats, and camels. Opisthorchis felineus is reported to be prevalent in countries such as Poland, Eastern Germany, and in parts of the former Soviet Union. Conditions such as cholangiocarcinoma and adenocarcinoma emanated from hyper-plastic activity of epithelial layer of the bile duct are associated with Opisthorchis viverrini and Clonorchis sinensis [39]. The adult flukes may occur in large numbers in bile ducts; and the large flask-shaped operculated eggs shed in feces and hatch after ingested by snail (intermediate host). Infection occurs through eating undercooked or raw fish, and the metacercariae reach the liver by direct migration up the bile duct. Raw fish dishes are a dietary habit in all countries where these flukes are commonly found [40]. Infection 
may increase the risk of cholangiocarcinoma, otherwise clinical manifestations are rare.

Fascioliasis: The Third Global Meeting of the Partners for Parasite Control held in WHO Headquarters Geneva in November 2004 has agreed on fascioliasis to be important foodborne helminths [38]. Fasciolosis is an emerging plant borne zoonotic disease reported from Northern provinces of Iran around Caspian Sea, high altitude Andean rural regions of Bolivia [41], Nile Delta region of Egypt, and Central provinces of Vietnam [42].

The two species namely, Fasciola hepatica and Fasciola gigantica are liver fluke that cause fasciolosis [43]. Consumption of water or plants with adherent metacercaria [44] or juvenile forms [42] causes infection in humans. Approximately, 2.4-17 million human infections are recorded worldwide after WHO recognized fascioliasis as an emerging disease of humans $[8,45]$. Fasciola hepatica infects various host species, which is cosmopolitan in distribution; and its intermediate host (snail) is well adapted to a wide range of ecological niches [46].

Fasciola gigantica is restricted to South and East Asia, Middle East, and tropical regions of Africa due to reduced ability of the aquatic snail to invade new niches. Ingestion of watercress and other edible aquatic plants contaminated with encysted metacercariae is the most common transmission route. Clinical signs, such as weight loss, malaise, abdominal discomfort, and diarrhea occur due to migration of the immature flukes through the liver parenchyma [47].

Fasciolopsiasis: Fasciolopsiasis is another foodborne helminth caused by Fasciolopsis buski, 'giant intestinal fluke', which is found in small intestine of humans, and pigs. It is transmitted by consumption and handling of aquatic plants, water chestnut, water hyacinth, and water morning glory contaminated by metacercariae infected. Drinking or using untreated water, and peeling off contaminated plants using teeth are other risks for transmission of $F$. buski infection to humans. Pigs are the only common reservoir host harboring few flukes. Even though, there are reports of emergence of human fasciolopsiasis in Uttar Pradesh State of India, the high infection rate is recorded among people in Assam, North-East India [48,49]. The disease have clinical impacts including hemorrhage, abscesses, catarrhal inflammation, erosions of intestine, ulceration, abdominal pain, and yellow offensive stools [50].

Paragonimiasis: Paragonimiasis is an infection caused by Paragonimus westermani in the lower respiratory tract. The disease has a major socioeconomic importance in Asian countries such as Philippines, China, Japan, Korea, Thailand; and incidence reports from African countries including Cameroun, Liberia, Nigeria, and also in South America. The parasite is transmitted to humans and other mammals such as pigs, dogs, cats, fox, and other wild carnivores via snails to freshwater crab or crayfish, which act as second intermediate host. Globally, around 294 million people are at risk of infection with Paragonimus species. Ingestion of infective metacercariae together with raw or undercooked crustaceans infects humans [51].

Clinical symptoms of paragonimiosis sometimes may be mistaken with chronic tuberculosis, and is usually appearing to be asymptomatic in humans. Clinical features such as, cough, blood-tinged sputum, dysponea, eosinophilia, recurrent haemoptysis, and distressing chest pain are remarkable symptoms of pulmonary paragonimiosis that sometimes confused with those of tuberculosis. When the parasite is migrating to the brain (extra-pulmonary location), it causes brain cysticercosis, tumour, epilepsy or embolism. Control of the parasite is very difficult task but treating reservoir host (dogs and cats) and avoiding trends of eating raw, under-cooked or freshly pickled crabs or crayfish in endemic areas can help in controlling the parasite in humans [39].

\section{Public health impact and economic importance of foodborne zoonotic helminthes}

Zoonotic diseases are the diseases, which are naturally transmitted between animals and humans. Transmission may occur in a number of settings, ranging from indirect contact through food or drinking water to direct contact through occupational exposure on farms, during leisure pursuits or from pets. Foodborne parasitic infections have been recently identified as an important public health problem having considerable economic impact in terms of morbidity, loss of productivity and health care costs. Poor sanitation and traditional methods of food preparation accelerated the spread of foodborne trematode infections [52].

Globally, economic loss resulted from foodborne parasitic zoonoses is difficult to assess due to inadequate information on the prevalence and public health implication of parasitic zoonoses for most countries. However, significant economic losses caused by certain zoonoses have been estimated for some regions. For example, in Mexico, swine cysticercosis caused a loss of more than US\$ 17 million annually in hospitalization and treatment costs for humans with neurocysticercosis, which was more than one-half of the national investment in swine production. In addition in Latin America and Africa, porcine cysticercosis accounts for an economic loss of US\$164 million and 2 billion, respectively. According to prioritizing results on foodborne parasitic zoonoses within each country's complex economic and public health problems, diseases caused by Taenia solium ranked $1^{\text {st }}$ and Echinococcus granulosus and E. multilocularis ranked $3^{\text {rd }}$ and $4^{\text {th }}$, respectively. This finding showed that foodborne helminthiasis is contributing to economic losses both in human and animal populations worldwide [53].

\section{Prevention and Control}

Parasitic zoonoses can be controlled by applying mass chemotherapy approaches using parasitic drugs. Education of consumers, food handlers, and food producers particularly regarding basic hygiene, sufficient cooking, and avoiding cross-contamination have all had an impact on foodborne transmission of pathogens and remain imperative to food safety. Disinfection of drinking water, sewage treatment, pasteurization, freezing, retort canning, shellfish bed sanitation, and the use of anti-parasitic compounds have all reduced the prevalence of foodborne helminths and, consequently, foodborne parasitic diseases [54]. In developed countries, surveillance of foodborne disease has become a fundamental component of food safety systems [55]. Uncooked foods, including salads and vegetables should be properly washed and treated with vinegar before consumption [56].

It is important to strengthen veterinary services to enhance efforts to control infections in livestock. It is also necessary to develop legally enforceable safe slaughter and meat inspection capability, especially in developing countries, and to assign foodborne infections higher priority in food safety programs. Immunization of food animals against foodborne parasites in certain conditions is very limited because there is generally a lack of vaccines for foodborne parasites. However, vaccination of pigs against $T$. solium, the parasite considered of greatest global importance according to WHO/FAO [57], has shown 
considerable promise [58] including in field trials [59]. Similarly, field trials of a vaccine against $E$. granulosus in sheep have provided promising results, and may be the method of choice to control these important foodborne cestode infections in livestock [60].

\section{Conclusion}

Foodborne illnesses comprise a variety of infectious and noninfectious diseases, which are responsible for significant proportion of morbidity and mortality as well as economic losses. Due to various reasons, it is difficult to determine the exact mortality and economic loss associated with foodborne diseases. It is also revealed that, both developing and developed countries share the global burden of helminthic foodborne diseases. More than one-quarter of the world's population is estimated to be chronically infected with intestinal parasites and majority of these infected people live in developing countries. Many parasitic infections are associated with overcrowding, poor sanitation, contaminated food and water, under nutrition, and other poverty-related factors. Therefore, hygienic handling of food and water, proper sanitation of equipments and avoidance of surface contamination as well as taking actions for interrupting survival of the harmful stages of parasites that is pathogenic to man is mandatory. Countries should keep pace with population growth; minimize migration from rural to urban areas and the demand for clean, safe drinking water and proper sanitation should be well implemented.

\section{Conflict of Interest}

None

\section{Source of Funding/Grant}

None

\section{References}

1. Helms M, Vastrup P, Gerner-Smidt P, Molbak K (2003) Short and long term mortality associated with foodborne bacterial gastrointestinal infections: registry based study. Br Med J (Clinical Research Ed.) 326: 326-357.

2. WHO (2015) WHO estimates of the global burden of foodborne diseases: foodborne disease burden epidemiology reference group 2007-2015. Geneva, Switzerland.

3. Linscott AJ (2011) Food-borne illnesses. Clin Microbiol News 33: 41-45.

4. Chala B (2013) A retrospective analysis of the results of five year (2005-2009) parasitological examination for common intestinal parasites from Bale-Robe Health Center, Robe Town, Southeastern Ethiopia, ISRN. Parasitol 2013: 1-7.

5. Prasad KJ (2010) Emerging and re-emerging parasitic diseases. J Int Med Sci Acad 23: 45-50.

6. Torgerson PR, Devleesschauwer B, Praet N, Speybroeck N, Willingham AL, et al. (2015) World Health Organization Estimates of the Global and Regional Disease Burden of 11 Foodborne Parasitic Diseases, 2010: A Data Synthesis. PLoS Med 12: e1001920.

7. Torgerson PR, Macpherson CN (2011) The socioeconomic burden of parasitic zoonoses: global trends. Vet Parasitol 182: 79-95.

8. Slifko TR, Smith HV, Rose JB (2000) Emerging parasite zoonoses associated with water and food. Int J Parasitol 30: 1379-1393.

9. Cox FE (2002) History of human parasitology. Clin Microbiol Rev 15: 595-612.

10. Macpherson CN (2005) Human behaviour and the epidemiology of parasitic zoonoses. Int J Parasitol 35: 1319-1331.
11. Chai JY, Murrell KD, Lymbery AJ (2005) Fish-borne parasitic zoonoses: status and issues. Int J Parasitol 35: 1233-1254.

12. Vuylsteke P, Bertrand C, Verhoef GE, Vandenberghe P (2004) Case of megaloblastic anemia caused by intestinal taeniasis. Ann Hematol 83: 487-488.

13. Macpherson CN, Gottstein B, Geerts S (2000) Parasitic food-borne and water-borne zoonoses. Rev Sci Tech Off Int Epiz 19: 240-258.

14. Moro P, Schantz PM (2009) Echinococcosis: a review. Int J Infect Dis 13: 125-133.

15. Das SS, Kumar D, Sreekrishnan R (2003) Hydatidosis in animals and man, In: Helminthology in India, Sood ML (Ed), International Book Distributors, Dehradun, India.

16. Eckert J, Deplazes P (2004) Biological, epidemiological, and clinical aspects of echinococcosis, a zoonosis of increasing concern. Clin Microbiol Rev 17: 107-35.

17. Pal M, Dutta JB (2013) Echinococcosis - An Emerging and Re-Emerging Cyclozoonosis of Global Importance. Int J Liv Res 3: 5-13.

18. Sotelo J (2003) Neurocysticercosis: eradication of cysticercosis is an attainable goal. Br Med J 326: 511-512.

19. Uga S, Hoa NT, Noda S, Moji K, Cong L, et al. (2009) Parasite egg contamination of vegetables from a suburban market in Hanoi, Vietnam. Nepal Med College J 11: 75-78.

20. Willingham AL, Wu HW, Conlan J, Satrija F (2010) Combating Taenia solium cysticercosis in Southeast Asia: an opportunity for improving human health and livestock production. Adv Parasitol 72: 235-266.

21. Dorny P, Somers R, Cam T, Dang T, Nguyen V, et al. (2004) Cysticercosis in Cambodia, Lao PDR and Vietnam. Southeast Asian J Trop Med Public Hlth 35: 223-226.

22. Garcia HH, Gonzalez AE, Evans CA, Gilman RH (2003) Taenia solium cysticercosis. Lancet 362: 547-556.

23. Kraft R (2007) Cysticercosis: An Emerging Parasitic Disease. Am Family Phys 76: 91-96.

24. Prasad KN, Prasad A, Verma A, Singh AK (2008) Human cysticercosis and Indian scenario: A review. J Biosci 33: 571-582.

25. Murrell KD (2005) Epidemiology of taeniosis and cysticercosis. In: Murrell KD (Ed.), WHO/FAO/OIE Guidelines for the Surveillance, Prevention and Control of Taeniosis/Cysticercosis. WHO for Animal Health (OIE), Paris, pp. 27-43.

26. Jongwutiwes S, Putaporntip C, Chantachum N, Sampatanukul P (2004) Jejunal perforation caused by morphologically abnormal Taenia saginata infection. J Infect 49: 324-328.

27. Han ET, Guk SM, Kim JL, Jeong HJ, Kim SN, et al. (2003) Detection of parasite eggs from archaeological excavations in the Republic of Korea. Mem Inst Oswaldo Cruz 98: 123-126.

28. Robertson LJ, Gjerde B (2000) Isolation and enumeration of Giardia cysts, Cryptosporidium oocysts, and Ascaris eggs from fruits and vegetables. J Food Prot 63: 775-778.

29. Angesom H, Pal M (2016) Ascariasis: Public health importance and its status in Ethiopia. Air Water Borne Dis 5:124-128.

30. Chitwood MB, Vaalasquez C, Salazar NG (1964) The physiological changes in a species of Capillaria causing a fatal case of human intestinal capillariasis. In: Proc1stInt Congr Parasitol.

31. Pal M, Seid H, Giro B, Wakjira B, Abdo J, et al. (2013) GnathostomiasisAn emerging nematodal zoonotic disease. Inter J Liv Res 3: 12-17.

32. Waikagul J, Chamacho SD (2007) Gnathostomiasis. Food-Borne Parasitol Zoonoses 235-261.

33. Herman JS, Chiodini PL (2009) Gnathostomiasis, another emerging imported Disease. Clin Infect Dis 22: 484-92.

34. Pozio E, Hoberg E, La Rosa G, Zarlenga DS (2009) Molecular taxonomy, phylogeny and biogeography of nematodes belonging to the Trichinella genus. Infec Gen Evol 9: 606-616.

35. Shimshony A (2009) Trichinellosis: a re-emerging helminthic zoonosis. Infect Dis News.

36. Pozio E, Murrell KD (2006) Systematics and epidemiology of Trichinella. Adv Parasitol 63: 367-439. 
Citation: Pal M, Ayele Y, Hadush A, Kundu P, Jadhav VJ (2018) Public Health Significance of Foodborne Helminthiasis: A Systematic Review. J

Page 6 of 6

37. Pozio E (2007) Taxonomy, biology and epidemiology of Trichinella parasites. In: Dupouy-Camet J, Murrell KD (Eds.), FAO/WHO/OIE Guidelines for the Surveillance, Management, Prevention and Control of Trichinellosis. OIE Publisher, Paris, France, pp. 1-35.

38. Brockelman WY, Upatham ES, Viyanant V, Hirunkraks A (1987) Measurement of incidence of the human live fluke, Opisthorchis viverrini in northeast Thailand. Trans Royal Soc Trop Med Hyg 81: 327-335.

39. Liu Q, Wei F, Liu W, Yang S, Zhang X et al. (2008) Paragonimiasis: an important food-borne zoonosis in China. Trends Parasitol 24: 318-323.

40. Anonymous (2004) Editorial: Thinking beyond deworming. Lancet 364: 1993-1994.

41. Moghaddam AS, Massound J, Mahmoodi M, Mahvi AH, Periago MV, et al. (2004) Human and animal fascioliasis in Mazandarin province, northern Iran. Parasitol Res 94: 61-69.

42. De NV, Murrell KD, Congle D, Cam PD, Chau V, et al. (2003) The foodborne trematode zoonoses of Vietnam. Southeast Asian J Trop Med Pub Health 34: 12-34.

43. Pal M, Mukarim A, Mesfin Z (2014) Growing significance of fascioliasis as an emerging zoonosis. Eth Int J Multid Res 1:10-13.

44. Ashrafi K, Valero MA, Massoud J, Sobhani A, Solaymani-Mohammadi S, et al. (2006) Plant-borne human contamination by fascioliasis. Am J Trop Med Hyg 75: 295-302.

45. Taira N, Yoshifuji H, Boray JC (1997) Zoonotic potential of infection with Fasciola spp. by consumption of freshly prepared raw liver containing immature flukes. Int J Parasitol 27: 775-779.

46. Bhatia BB, Pathak KML, Banerjee DP (2006) A Textbook of Veterinary Parasitology, 2nd Ed, Kalyani Publishers, New Delhi, India.

47. Le TH, De NV, Agatsuma T, Blair D, Vercruysse J, et al. (2007) Molecular confirmation that Fasciola gigantica can undertake aberrant migrations in human hosts. J Clin Microbiol 45: 648-650.

48. Bhatti HS, Malla N, Mahajan RC, Sehgal R (2000) Fasciolopsiasis, a reemerging infection in Azamgarh (Uttar Pradesh). Indian J Pathol Microbiol 43: 73-76.
49. Muralidhar S, Srivastava I, Aggarwal P, Jain N, Sharma DK, et al. (2002) Fasciolopsiasis, a persistent problem in eastern U.P: A case report. Indian J Pathol Microbiol 43: 69-71.

50. Mas-Coma S, Barques MD, Valero MA (2005) Fascioliasis and other plant-borne trematode zoonoses. Int J Parasitol 35: 1255-1278.

51. Vijayan VK (2009) Parasitic lung infections. Current Opinion Pulmonary Med 15: 274-282.

52. Phan VT, Ersboll VK, Nguyen H, Madsen A, Dalsgaard A, et al. (2010) Farm-level risk factors for fish-borne zoonotic trematode infection in integrated small-scale fish farms in North Vietnam. Plos Neglected Trop Dis 4: 742 .

53. Carabin H, Budke CM, Cowan LD, Willingham AL, Torgerson PR, et al. (2005) Methods for assessing the burden of parasitic zoonoses: echinococcosis and cysticercosis. Trends Parasitol 21: 327-333.

54. Pal M (2007) Zoonoses. 2nd Ed. Satyam Publishers, Jaipur, India.

55. Gervelmeyer A (2008) Developing the community reporting system for food borne, outbreaks. Euro Surveill 13: 45.

56. Escobedo MA, Homedes N, Aldana V, Alt KV, Serrano BN, et al. (2003) Assessment of parasitic diseases in children in five communities in the border region of Far West Texas. Texas Department of Health Office of border health (TDH), pp 1-20.

57. Robertson LJ (2013) Have foodborne parasites finally become a global concern? Trends Parasitol 29: 101-103.

58. Sciutto E (2013) Development of the S3PVAC vaccine against porcine Taenia solium cysticercosis: a historical review. J Parasitol 99: 686-692.

59. Assana E (2010) Elimination of Taenia solium transmission to pigs in a field trial of the TSOL18 vaccine in Cameroon. Int J Parasitol 40: 515-519.

60. Larrieu E (2013) Pilot field trial of the EG95 vaccine against ovine cystic echinococcosis in Rio Negro, Argentina: early impact and preliminary data. Acta Trop 127: 143-151. 